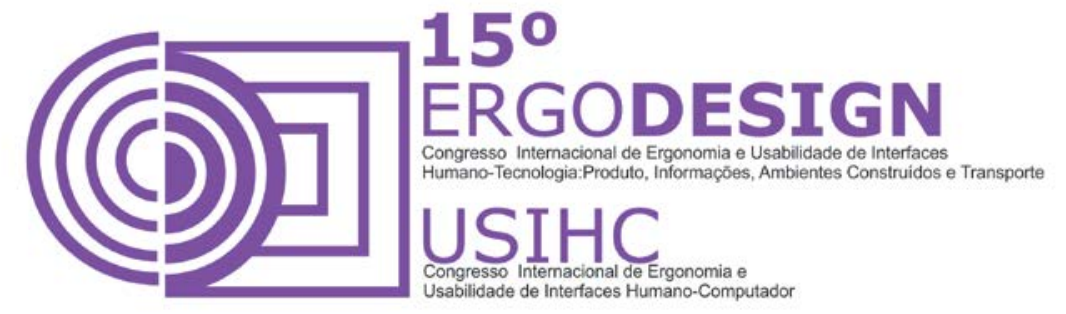

\title{
ANÁLISE DE CONCEITOS PARA CRIAÇÃO DE UM MODELO CONCEITUAL PARA O USO DE GAMIFICAÇÃO COMO AUXÍLIO AO DESENVOLVIMENTO COGNITIVO DE CRIANÇAS COM TDAH
}

\author{
CONCEPTS OF ANALYSIS FOR CREATING A CONCEPTUAL \\ FRAMEWORK FOR THE USE OF GAMIFICATION AS AID TO THE \\ COGNITIVE DEVELOPMENT OF CHILDREN WITH ADHD
}

\author{
OLIVEIRA, Izautino (1); \\ (1) CESAR Edu, mestrando \\ e-mail:izautinooliveira@gmail.com
}

\begin{abstract}
RESUMO
O Transtorno do Déficit de Atenção e Hiperatividade (TDAH) é uma doença de alta prevalência em crianças na fase escolar. O TDAH é reconhecido como uma condição importante, pelo forte impacto funcional e sendo caracterizado pela persistência de sintomas, superior a seis meses e observado em pelo menos dois contextos, casa e escola. Este artigo se propôs a realizar uma investigação sobre o TDAH, Terapia Cognitivo-Comportamental (TCC) e como a utilizar a mecânica a Gamificação para criar uma metodologia docente diferenciada, centrada nas características específicas das crianças com este déficit, agindo como modelo para auxiliar o desenvolvimento cognitivo de seus portadores.
\end{abstract}

Palavras chaves: Gamificação, TDAH, Jogos, Desenvolvimento Cognitivo.

\section{ABSTRACT}

The Attention Deficit Hyperactivity Disorder (ADHD) is a highly prevalent in school-age disease. ADHD is recognized as an important condition for strong functional impact and being characterized by the persistence of symptoms, more than six months and observed in at least two contexts, home and school. This paper set out to conduct research on $A D H D$, cognitive-behavioral therapy (CBT) and how to use the mechanical gamification to create a differentiated teaching methodology, focused on the specific characteristics of children with this deficit, acting as a model to assist the development cognitive of their bearers.

Keywords: Gamification, ADHD, Games, Cognitive Development. 


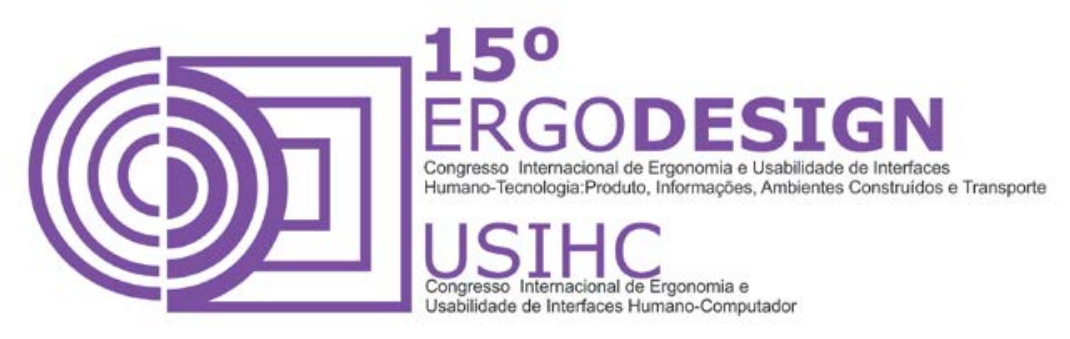

\section{INTRODUÇÃO}

O TDAH é o transtorno comportamental infantil frequentemente diagnosticado, afeta cerca de 3 a $6 \%$ de crianças em fase escolar, onde de $30 \%$ a $50 \%$ dos casos persistem até a idade adulta (Azambuja, 2013). As crianças diagnosticadas com o transtorno apresentam alguns problemas na escolar: maior probabilidade de repetência, maior evasão escolar, baixo rendimento, na maioria dos casos, a criança acaba adquirindo um bloqueio na aprendizagem ou em questões comportamentais. As causas desse transtorno são, em grande parte, desconhecidas, e envolvem fatores genéticos, neurobiológicos e até mesmo ambientais. Esse transtorno, nos casos mais sérios é tratado com medicamentos, mesmo que os medicamentos tragam efeitos positivos de curto prazo, no médio e longo prazo se mostram insuficientes para atender às necessidades da criança, especialmente com organização, planejamento, entre outros (Smolka, 1993).

Toda teoria por trás da Terapia Cognitivo-Comportamental (TCC) ajuda a criança com TDAH perceber que ela possui capacidade para modificar o seu comportamento e pensamento, como também desenvolver algumas estratégias de autocontrole. O tratamento consiste em várias fases, dentre algumas a psicoeducação (tornar o paciente ciente dos sintomas do transtorno), a psicoterapia em si e intervenções no meio ambiente (Camargos, 2005). É no estágio de intervenção que conseguimos aliar o uso da Gamificação, ou seja, a utilização de elementos dos jogos (mecânicas, estratégias, pensamentos) fora do contexto dos jogos, com a finalidade de motivar o portador à ação.

\section{O TDAH}

O Transtorno de Déficit de Atenção e Hiperatividade é um transtorno caracterizado pela presença de desatenção, hiperatividade e impulsividade. O TDAH causa grande impacto na vida de seus portadores, principalmente em crianças. Estas apresentam dificuldades em se relacionar com os outros, são instáveis e tumultuadas, acompanhadas de um baixo desempenho escolar, o que acaba por acarretar enormes prejuízos no ambiente familiar e social (Mesquita et al, 2009).

A avaliação psicológica e o diagnóstico do Transtorno de Déficit de Atenção e Hiperatividade envolvem um processo delicado, o qual demanda de um profissional com um bom conhecimento teórico e, sem dúvida, muita reflexão. As escolas, a cada dia que passa se envolvem em uma tendência de explicar o mau desempenho de seus alunos pela presença do TDAH. Ainda que em muitos casos o diagnóstico realizado na escola possa estar equivocado, o Transtorno de Déficit de Atenção e Hiperatividade é responsável por uma boa parcela dos problemas escolares e sociais, tendo em vista que ele, independentemente da associação com a hiperatividade, compromete significativamente o desempenho escolar, pois prejudica uma condição indispensável para a aprendizagem como um todo (OMS, 1993).

Uma criança pode estar inquieta ou distraída por muitos motivos, e não necessariamente devido a um transtorno. A inquietação pode ser indicativa de uma inteligência ativa, 


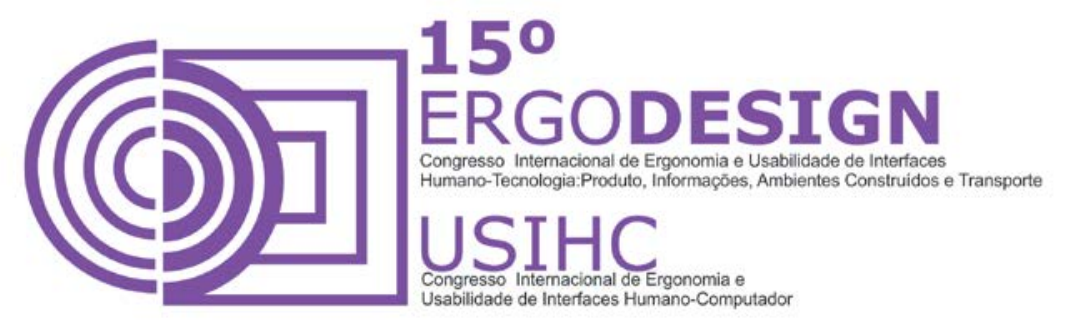

questionadora, que deve ser adequadamente estimulada nos meios familiar e escolar. Estar no "mundo da lua" pode simplesmente ser um artifício inconsciente de mobilizar a atenção para os múltiplos problemas emocionais e de aprendizagem, que merecem cuidados objetivos (Graeff; Vaz, 2008).

Hoje, já se sabe que a área do cérebro afetada pelo transtorno é a região frontal, responsável pela atenção, organização, funções executivas e também o sistema emocional de recompensa. O que acontece é uma alteração no funcionamento do sistema de substâncias químicas, neurotransmissoras, principalmente dopamina e noradrenalina que passam informação entre os neurônios (Mattos et al, 2012).

As intervenções presentes na Terapia Comportamental-Cognitiva podem ser feitas através de jogos. Os jogos permitem que a criança com TDAH liberte a tensão, frustração, insegurança e até mesmo a agressividade, medo e a confusão, tudo isso sem que a criança se dê conta que tem todos esses sentimentos guardados. Deste modo, os jogos podem ser vistos como recursos que auxiliam a aprendizagem. A hiperatividade dificulta o desenvolvimento de um comportamento social adequado em uma criança hiperativa e através dos jogos ela pode aprimorar seu senso de respeito às normas grupais e sociais. Em uma situação de jogo, a criança se expõe a estímulos variados e aprende a se comportar de forma diferenciada frente às estimulações, conforme as regras e as contingências envolvidas (Ferreira, 2009).

\section{OS JOGOS}

Existem bilhões de jogadores que passam milhares de horas em ambientes virtuais resolvendo desafios. Essa quantidade cada vez maior de jogadores é entendida pelo fato de que em ambientes virtuais é encontrada uma satisfação para viver. Jogadores de videogame reconhecem o valor da prática prolongada e desenvolvem qualidades pessoais, tais como persistência, criatividade e resiliência. Para entender o que cativa um jogador é importante descobrir suas motivações e de que formas os jogos trabalham seus desejos e geram novas necessidades. A importância da experiência de um jogo depende de quanto interesse ele pode gerar. Criar e manter o interesse dos jogadores é a maneira de gerir a sua motivação. Sua motivação é o fator que irá determinar se um jogador vai continuar a jogar depois de alguns minutos, bem como quanto tempo ele vai jogar e se ele vai terminar o jogo (Sena; Kerr, 2012).

O desafio ao aliar Gamificação e TDAH está relacionado à motivação do portador. As crianças que apresentam o quadro têm imensa dificuldade em começar uma atividade e terminá-la sem antes já iniciar outra, deixando as outras esquecidas. Ainda que os videogames sejam uma ferramenta perspicaz, a problemática da desatenção precisa de uma série de recursos para manter um longo diálogo com o jogador.

A Gamificação é utilizada como tentativa de aproveitar o poder motivador dos jogos e aplicá-lo aos problemas do mundo real, uma vez que compartilha dos elementos e do design dos jogos para atingir propósitos em comum como, por exemplo, lançar desafios, usar estratégias, obter pontos para atingir determinados objetivos e liberar acesso a itens bloqueados, conquistar espaço, ganhar visibilidade e recompensas, medalhas, prêmios, entre outros (Lee; Hammer, 2011). As técnicas usadas na Gamificação estimulam o engajamento e da criança nas atividades diárias, através de funções psicológicas, emocionais e seu poder social. Tal processo 


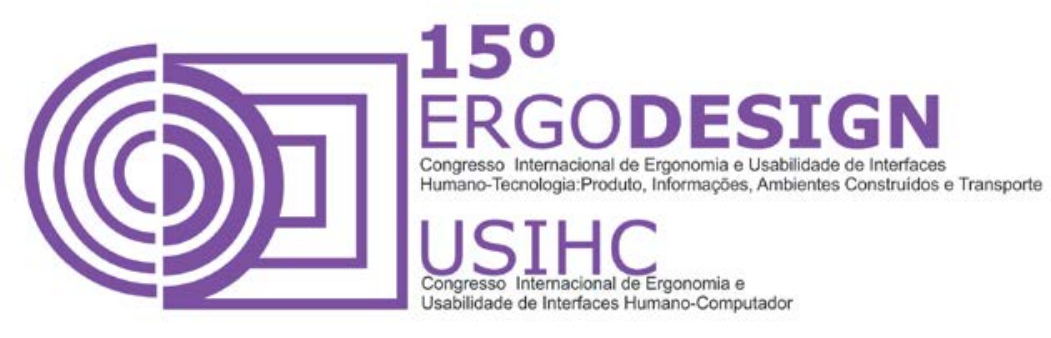

pode ser explicado do ponto de vista neurobiológico: no cérebro de crianças com TDAH, ocorre uma maior reabsorção de neurotransmissores como dopamina e noradrenalina, responsáveis por sensações de maior satisfação e saciedade. Quando se joga, e principalmente quando se ganha, há uma liberação desses neurotransmissores.

\section{O MODELO}

Gamificação, por definição, seria aplicação de design e princípios de jogos para ambientes de fora do jogo. Por outro lado, a Gamificação pode ser vista como o uso de atributos de jogo para dirigir o comportamento dos jogadores em outro contexto. No entanto, Gamificação e aprendizagem estão relacionadas porque ambos tentam aspectos de motivação para conseguir algo mais. A Gamificação faz isso através de um amplo conjunto de ferramentas (por exemplo, mecânica de jogo, game design, psicologia do jogo, etc.).

Ao associar os conceitos, Gamificação e TDAH, a família e escola começam a direcionar a aprendizagem numa perspectiva inclusiva em prol do completo aprendizado e do bem-estar de todos os envolvidos. Entende-se, portanto, que a criança com TDAH possui potencialidade para desenvolver suas capacidades de aprendizagem e inserção na sociedade.

\subsection{Atores e Pontos Chave do Modelo}

O ser humano apresenta a tendência de aprender mais facilmente um corpo de conhecimentos quando ele é apresentado a partir de suas ideias mais gerais e mais inclusivas e se desdobrando para as ideias mais específicas e menos inclusivas (Ausubel, 2003). O modelo de referência é proposto como uma estratégia facilitadora de aprendizagem visando auxiliar no desenvolvimento cognitivo da criança com TDAH. O portador do TDAH precisa ter um acompanhamento especial, já que não consegue conter seus instintos. Os pais e professores tem papel fundamental na aprendizagem da criança com TDAH, o mesmo deve estar preparado para utilizar todos os tipos de recursos disponíveis, tendo assim maiores estratégias a seu dispor. Utilizando a mecânica da Gamificação, os educadores poderão estimular as crianças à aprendizagem.

Ao introduzir a Gamificação no seu dia a dia, a criança com TDAH passa por uma fase de adaptação e reconhecimento, sendo interessante que ela o faça com auxílio da família, explorando todas as suas possibilidades, pois jogar implica em ação. Através da Gamificação a criança é estimulada a se desafiar e aprender com isso, fazendo novas descobertas. Quando a criança com TDAH vive isoladamente, a Gamificação pode estimulá-las ao convívio em grupo, por isso é extremamente importante que os educadores saibam da importância da atividade lúdica para o desenvolvimento da criança. . A dinâmica rápida proporciona à criança com TDAH a capacidade de desenvolver suas funções cognitivas. Assim, para permitir um envolvimento significativo e mudança de comportamento é necessário conhecer a criança com o transtorno em seu contexto, bem como compreender os detalhes, exemplos e ideias da mecânica por trás da Gamificação. Abaixo, os pontos chave para o modelo:

Passo 1: Criança 


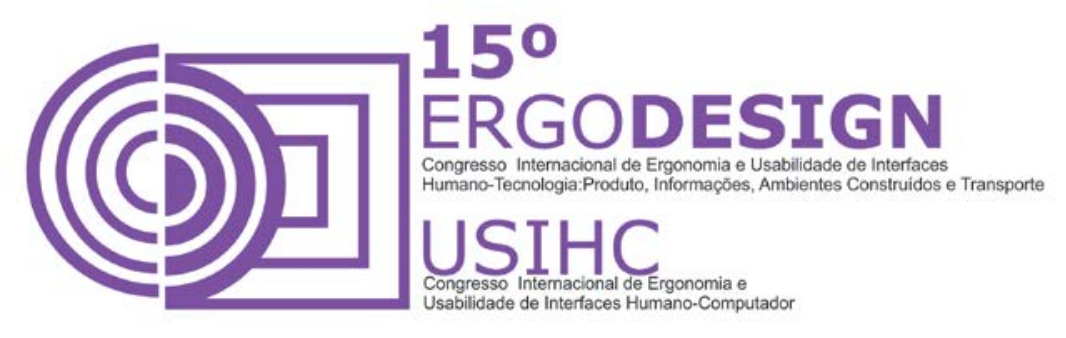

O primeiro passo para a abordagem de desenvolvimento do modelo é centrado na criança, compreende-la em seu contexto, casa e escola, por exemplo. O sucesso de seus esforços depende dessa compreensão clara.

Passo 2: Objetivo

O próximo passo é definir o objetivo. Esta etapa envolve a compreensão do cenário atual, identificando o resultado desejado e estabelecendo um objetivo adequado para a criança.

Passo 3: Motivação

Há dois tipos gerais de motivações: intrínsecos e extrínsecos. Intrínseca refere-se às motivações internas, tais como autonomia, domínio e significado. Extrínseca refere-se a técnicas motivacionais externos, tais como dinheiro, troféus, etc.

\section{Passo 4: Aplique mecânica}

Com uma compreensão clara da criança, o objetivo, e a teoria por trás motivação, é hora de aplicar a mecânica de jogo, criar regras do jogo, e formar um laço engajamento. Mecânica de jogo refere-se aos elementos que irão interagir com a criança. Aqui está uma lista de mecânica de jogo que são relevantes ao modelo:

- Pontos: Pontos são métricas de contagem individuais. Esta é a forma como o sistema mantém a contagem das ações da criança com TDAH pertencentes aos comportamentos-alvo na estratégia global.

- Badges: Uma vez que a criança com o transtorno tenha acumulado um certo número de pontos, que podem ser atribuídas distintivos. Badges são uma forma de conquista da criança. Eles proporcionam o reforço positivo para o comportamento alvo.

- Relacionamentos: Os relacionamentos são a mecânica do jogo baseado na condução da motivacional de conexão. Os seres humanos são seres sociais e as relações têm um forte efeito sobre a forma como se sentem e o que fazem.

- Desafio é uma poderosa mecânica de jogo para motivar as crianças com TDAH à ação, especialmente se eles acreditam que estão trabalhando para conseguir algo grande, algo inspirador, e algo maior que elas mesmas.

O sucesso na aprendizagem de crianças com TDAH exige uma combinação de intervenções e acompanhamento. Com o auxílio da Gamificação, elas podem, perfeitamente, desenvolver suas habilidades cognitivas.

\subsection{Estrutura do Modelo}

O modelo de referência pode ser utilizado como uma linguagem gráfica para a descrição e comunicação de conceitos e de seus relacionamentos. Com origem na aprendizagem através da Gamificação, tem como principal objetivo explicitar a relação entre um grupo de conceitos com o uso de proposições, que representam as unidades fundamentais do conhecimento, as unidades semânticas que compõem a estrutura cognitiva. Em geral, dentro do ambiente gráfico do modelo, os conceitos estão representados por círculos e as relações entre estes conceitos 


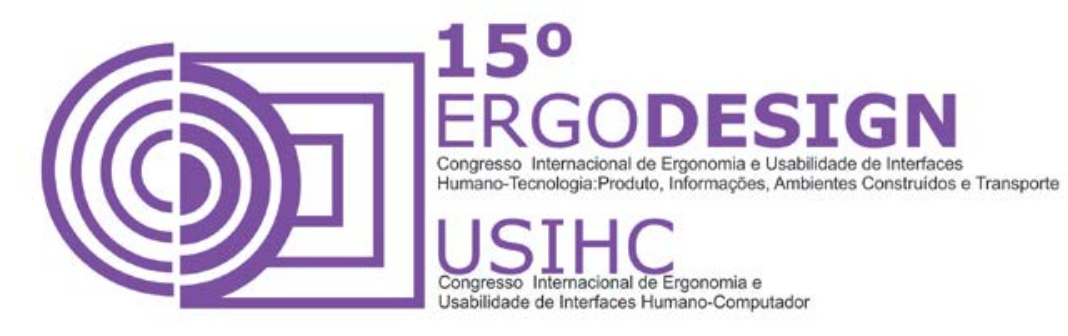

são representadas por linhas que conectam cada conceito. As linhas possuem palavras associadas e são elas que descrevem a natureza de cada relação (Figura 1).

Primeiramente é necessário analisar os conceitos importantes. Após a reflexão sobre os conceitos, é preciso observar as ligações que se entende existir entre esses conceitos de forma independente de ordem. Cada ator envolvido no aprendizado tem, ao se deparar com o modelo, autonomia para trilhar um caminho próprio na construção de seu conhecimento.

O modelo pode ser utilizado no contexto educacional e familiar para esclarecer e descrever as ideias que professores e pais têm sobre TDAH. A vantagem do uso deste tipo de representação do conhecimento é que o modelo de referência, por sua natureza gráfica, é mais eficiente para a "visualização" do conhecimento, ou seja, possibilita uma visão abrangente das relações entre cada um dos conceitos. Além disso, entende-se que a construção e interpretação do modelo exige um esforço cognitivo menor do que, por exemplo, para a construção e interpretação em forma de texto.

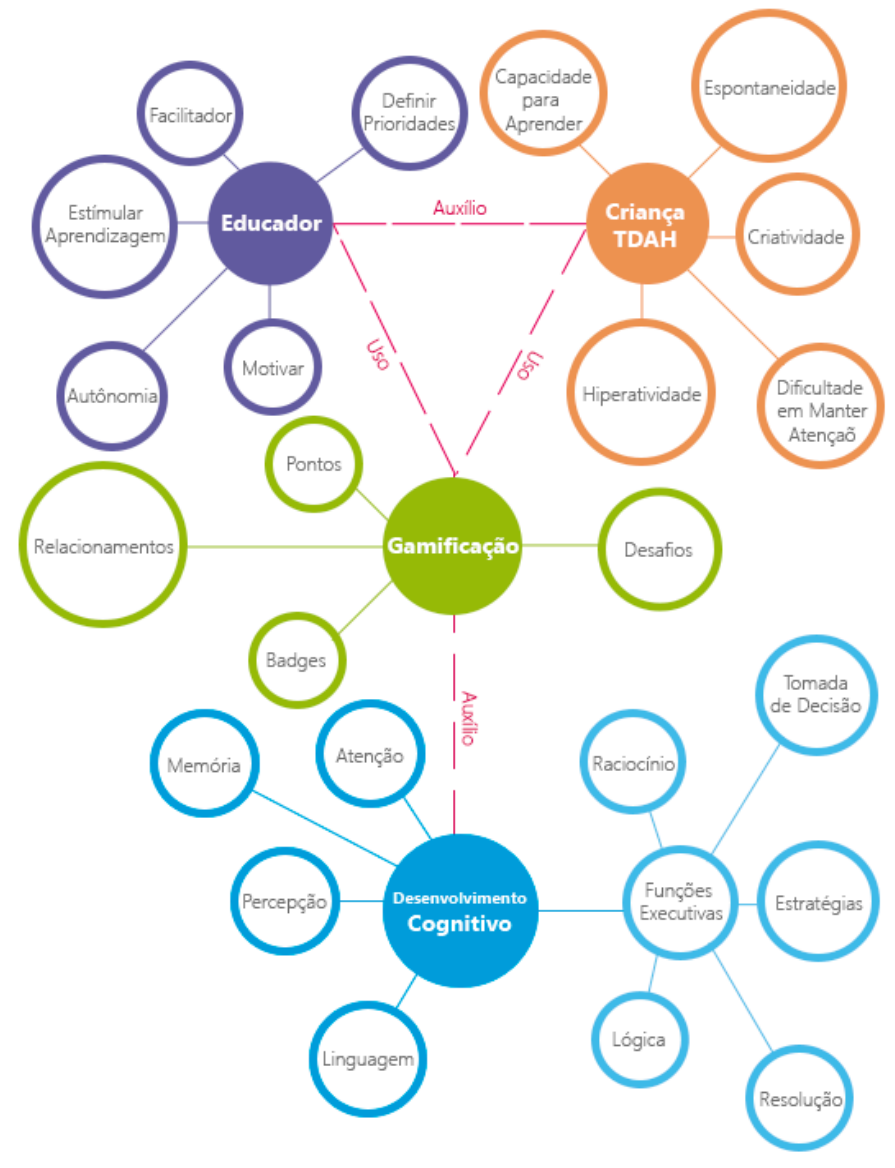




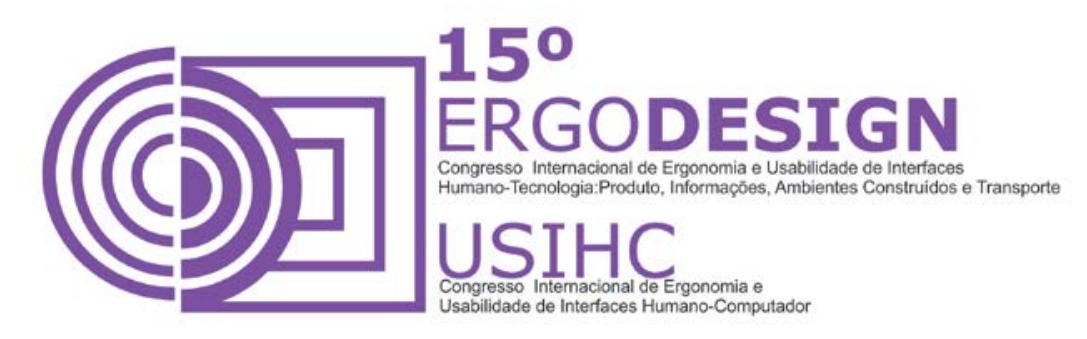

Figura 1 - Modelo Gráfico para Uso da Gamificação como Auxílio ao Desenvolvimento de Crianças com TDAH

\section{CONSIDERAÇÕES FINAIS}

Detectado o transtorno através de um profissional, este deve dar toda a orientação necessária para os pais. É no âmbito familiar que a criança buscará mais apoio, portanto os pais devem estar preparados. Muitos pais imaginam que a escola tem algum tipo de atendimento próprio para esses alunos hiperativos e desatentos, porém, essas crianças têm um potencial de aprendizagem igual ao de crianças normais. Elas precisam desse convívio social com colegas de mesma idade e também aprender a lidar com regras, pois de certa forma a escola representa, em pequena escala, a sociedade em que irão viver.

As intervenções, presentes no Tratamento Comportamental-Cognitivo fundidas com a mecânica da Gamificação, no ambiente escolar ou em casa auxiliam o desenvolvimento cognitivo visando alcançar o sucesso.

É perceptível que crianças portadoras de TDAH se adaptam melhor à Gamificação, uma vez que vão de encontro aos seus interesses e necessidades pessoais. Ou seja, a utilização de modelo de referência proposto age como instrumento educacional, e se caracteriza como subsídio a criança com TDAH e aos educadores nos significados dos processos de ensino/aprendizagem. $O$ modelo tem por finalidade conceber relações significativas entre os conceitos e as maneiras de proposições e auxiliar no desenvolvimento cognitivo destas crianças, através da Gamificação.

\section{BIBLIOGRAFIAS BIBLIOGRÁFICAS}

Roberta Azambuja. Diagnóstico Da Hiperatividade Exige Pelo Menos Seis Sintomas, 2013.

Smolka, A.L.B. A Criança Na Fase Inicial Da Escrita: A Alfabetização Como Processo Discursivo, Ed. Da Universidade Estadual De Campinas, 1993. 


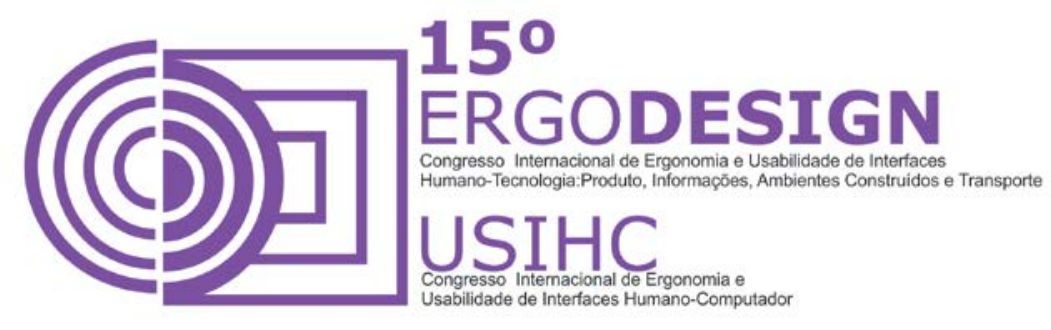

Camargos Jr., Walter Et Al. Transtornos Invasivos Do Desenvolvimento: $3^{\circ}$ Milênio. Brasília: Corde, 2005.

Cíntia Machado de Mesquita, Patrícia Ribeiro Porto, Bernard Pimentel Rangé, Paula Rui Ventura. Terapia Cognitivo-Comportamental e o Tdah Subtipo Desatento: Uma Área Inexplorada, 2009.

Organização Mundial De Saúde. Classificação E Transtornos Mentais E De Comportamento Da Cid10: Descrições Clínicas E Diretrizes Diagnósticas. Porto Alegre: Editora Artes Médicas, 1993.

Graeff, Rodrigo Linck; Vaz, Cícero E. Avaliação E Diagnóstico Do Transtorno De Déficit De Atenção E Hiperatividade (TDAH), 2008.

Prof. Dr. Paulo Mattos; Prof. Dr. Luis Augusto Rohde; Guilherme Polanczyk. TDAH: Questionários e escalas, 2012.

Ferreira, Paula Andréa Prata. Um Projeto Arquitetural Para Sistemas Neuropedagógicos Integrados, 2009.

Alexandre Sena; Dennis Kerr Coelho. Motivação dos Jogadores de Videogame - Uma breve visão sobre as Técnicas de Engajamento, 2012.

Lee, J. J. \& Hammer, J. Gamification in Education: What, How, Why Bother?, 2011.

Ausubel, D. Aquisição e retenção de conhecimentos: Uma perspectiva cognitiva, 2003. 